\title{
'Networks in the brain: from neurovascular coupling of the BOLD effect to brain functional architecture'
}

Luigi Barberini ${ }^{1,2^{*}}$ D, Francesco Marrosu ${ }^{1}$, Iole Tommasini Barbarossa ${ }^{3}$, Melania Melis ${ }^{3}$, Harman S. Suri ${ }^{4}$, Antonella Mandas ${ }^{1}$, Jashit S. Suri ${ }^{4}$, Antonella Balestrieri ${ }^{1,5}$, Michele Anzidei ${ }^{6}$ and Luca Saba ${ }^{1,5}$

\begin{abstract}
Background: Recently, many academic research groups focused their attention on changes in human brain networks related to several kinds of pathologies and diseases. Generally speaking, Network Medicine promises to identify the principles to understand, at the molecular level, the human life complex system. We applied the theoretical approach of the Network Medicine mainly to brain diseases, testing the potentiality of the method to produce an early and more in-depth diagnostic process. However, the range of our topics and application of the Network Medicine spans from common neurological and neuropsychological spectrum disorders to other physiological systems or district, only apparently out of the action range of the neurological one. In the areas of Neurology and Neuropsychology we are studying brain networks in Tourette Syndrome [TS] and Multiple Sclerosis [MS]. Moreover, we are studying brain networking related to genotype alteration responsible for different gustatory stimuli processing in the brain. Further, we recently started to study infective diseases and immunological system.
\end{abstract}

Main body of the abstract: The analysis of brain networks is made feasible by the development and application of imaging acquisition methods based on the Magnetic Resonance Imaging and functional MRI, as well as the availability of innovative calculation tools from graph theory and complex dynamical systems. This paper highlights the Network Medicine concepts and the application of the functional connectivity for the brain networks description. We will describe all these methods with a reduced use of formulas, along with some technical tools available in the WEB to perform the calculations of the related parameters. We will briefly describe the freely available software CONN used for calculating different connectivity parameters. In all these studies, the networks characterisation is performed using indexes from metric and topology of the brain networks. The possibility of early recognition of the diseases by the identification of the alteration in the network's parameters may significantly improve patient outcome, with also significant social community benefits. We intend to illustrate a possible pipeline for the Networks Brain application to neuroradiologists involved in the diagnosis of complex diseases.

Short conclusion: The multidisciplinary approach is one of the essential characteristics of journals in the imaging sciences field; the issues and the methodologies examined in this paper could be of great interest to the reader's community. Furthermore, until today very few studies focused on the network medicine and connectivity explored in functional MRI. Hence this might have an additional value for the journal.

\footnotetext{
* Correspondence: barberini@unica.it

${ }^{1}$ Department of Medical Sciences and Public Health, University of Cagliari,

Cagliari, Italy

${ }^{2}$ Department of Nuclear Medicine, Universitary Hospital of Cagliari AOU,

Cagliari, Italy

Full list of author information is available at the end of the article
} 


\section{Background}

The brain is connected! The topology and the metric of these connections across Central Nervous System (CNS), and Peripheral Nervous System (PNS) reflect a holistic view of human functions and life. Modern Medicine needs to identify new paradigms for diagnostic, and therapeutic approaches, to consider human health differently. All the biological systems, regarded as fundamental constituents of living beings, are structured and connected. Indeed, the connection between the systems and the connection across the systems are present. Connections in humans can occur between different elementary units such as neurons, nephrons, cardiomyocyte, and so on, and among various tissues and organs, generally through a large variety of molecules. In this context, the problem is to identify the structures and the properties of these connections and to determine the mathematical laws describing their dynamic [1] . This approach may potentially answer to critical medical-related questions through the accurate description of the ways used by Biological Systems to perform the vital functions.

Characterization of the networks in and towards the brain may be useful to evaluate diseases alterations in other organs, hence to optimise early diagnostic tools and treatments. In this paper, we report some applications of brain networks analysis, based on the nuclear magnetic resonance imaging, for the assessment of several human pathological mechanisms. Magnetic resonance imaging may be considered as a relevant application tool for this innovative strategy for the brain functions evaluation.

\section{fMRI and neurovascular coupling}

Magnetic resonance technology may be used in brain imaging to realise structural images of the inner part of the brain and to quantify functional neuronal activity. The most diffused technique for neurons activity measurement is electroencephalography (EEG). However, EEG only allows for the investigation of the cortical neurons functions. Functional magnetic resonance imaging, or functional MRI (fMRI) [2], indirectly measures brain activity by detecting changes associated with local blood flow. It is a low sensitivity technique with signals dynamic spanning a broad temporal range. In fact, this technique relies on the fact that cerebral blood flow and neuronal activation are indirectly connected. When the need to perform a task activates a particular area of the brain, the blood flow toward that region increases to compensate the consumption of the oxygenated haemoglobin (oxyHb). Indeed, local blood flow in the brain is strictly related to oxygen $(\mathrm{O} 2)$ and carbon dioxide (CO2) concentrations in tissues due to neurons energy consumption. This mechanism leads to regional differences in cerebral blood flow (CBF). Therefore, the Region of Interest (ROI), activated by the task, and the ones with low activity levels, because not involved, will show different blood flow dynamic.

We can describe the basic physiology of the "coupling" between neuronal activity and vascular brain system as follows: when a specific region of the brain increases its activity, responding to a task submitted to the subject, then the vascular system submits oxygen rich blood to this brain area. The higher local consumption of oxygen from the local capillaries leads to a decrease in oxy $\mathrm{Hb}$, and an increase in local deoxygenated haemoglobin (deoxy- $\mathrm{Hb}$ ) concentration [3]. Following a time delay of a few seconds respect to the starting of the task elaboration, CBF increases as physiological reaction to deliver further oxyHb. This process causes an increased in the regional concentration of oxygenated haemoglobin due to the increase of CBF a few seconds after neuronal activation. In $\mathrm{T} 2 \%$ contrast weighted EPI, commonly used in fMRI, the unbalanced growth of oxygenated haemoglobin local concentration, compared to the local oxygen consumption, causes an enhanced MRI signal intensity [4, 5].

This rapid unbalance in local tissue oxygenation induces a regional loss of coherence in the spin motion. Hence we can reveal a local variation in the Macroscopic Magnetization M [6], detachable in the Magnetic Resonance Imaging using the $\mathrm{T} 2 *$ contrast weighted Echo Planar Images (EPI) sequences. The variation of the local paramagnetic properties produces the loss of spin coherence. Since deoxyHb is paramagnetic and $\mathrm{oxyHb}$ is diamagnetic, the variation of the ratio between these two concentrations causes local dephasing of protons and modification of the signal detected from the tissues involved (blood-oxygen-level-dependent signal BOLD). Contrast for this kind of MR images is realised using T2*weighted sequences with the EPI pulses scheme used to observe this change [7]. Typical intensity of EPI sequences is in the range of $1-5 \%$ of the standard MRI signal, depending on the strength of the applied field.

Unfortunately, there are several limitations for BOLD effect-based fMRI and the scientific community is still debating on this topic [8]. Nevertheless, it is always considered as an essential tool for functional imaging, even when compared to other novel emerging functional techniques, such as Arterial Spin Labelling (ASL) [6-11].

\section{Description of resting state experiments and default mode network}

The bold signal can be detected not only during tasks performance but also in the so-called rest condition.

We can define the resting state as the mental state when a person may be awake and alert, but not focused on any activity requiring concentration or attention. In this condition, it is possible to consider spontaneous BOLD signal fluctuation. These fluctuations can be related to functional and physiological connections 
between different brain areas. These fluctuations were demonstrated in fMRI dataset in 1993 [12], but only recently these non-deterministic BOLD signal oscillations have been analysed with different mathematical techniques for the detection of functional connections of inter-hemispheric pathways. The analysis of nondeterministic BOLD signal oscillations aims to study physiological, pathologic, ageing- and drug-related changes in spontaneous blood oxygenation noise structure. Low-frequency resting state BOLD signals (typically $<0.1 \mathrm{~Hz}$ ) reveal coherent and spontaneous fluctuations that delineate the functional architecture of the human brain [13] . These fluctuations can bring pieces of information about brain activity also in the state of the brain defined as "resting state". Resting state fMRI (rsfMRI) is a method used to evaluate the global and local brain activity occurring when a subject is not performing specific tasks [14]. Along with BOLD signals oscillations, also the connectivity between different brain areas is active in the resting state.

The brain regions co-activated in rest condition define the default mode network (DMN) or default state network [14]: they are active in the default mode state of the brain, (intrinsic functional connectivity). Areas of the DMN are known to have activity highly correlated and distinct from other areas of the brain. It is considered necessary for several self-referential functions including imagination, conscious awareness, and conceptual processing. In particular, this pattern is most commonly observed to be active when a person is not focused on the outside world. The brain status in resting state is similar to the daydreaming, as a sort of wakeful rest, or, in other words, in the condition of mind-wandering. The Default Mode Network is also active when the individual is thinking about others or himself, triggering the action of recalling the past, and to plan for the future. Therefore, such network is activated "by default" when a person is not involved in a particular task. Therefore, DMN network is deactivated when the brain is required to perform some task, as in the Executive Attention Network (EAN) experiments [15]. Although DMN was initially noticed to be deactivated, also for specific goaloriented tasks, sometimes referred to as task-negative network, it can be active in other goal-oriented tasks such as social working memory or autobiographical tasks. Moreover, in the network analysis is important the correlation "sign". DMN is negatively correlated with other networks in the brain such as attention networks. Interestingly, the researchers observed DMN abnormalities in several neuropsychological conditions, as in the attention deficit hyperactivity disorder (ADHD) [16]. For this reason, research on DMN in resting state is regarded as potentially valuable for the elucidation of brain disease mechanisms.
In summary, BOLD effect plays an important role also in the resting condition of brain activity, in the absence of an externally prompted task, because information of interest may usually be observed as oscillations, or spontaneous fluctuations in BOLD signals [17]. Resting state approach may be useful to explore the functional organisation in the brain and its alterations in neurological or psychiatric diseases. Over the last ten years, resting state functional connectivity research has revealed many networks, which are consistently found in healthy subjects, different stages of consciousness and across species, and represent specific patterns of synchronous activity. In particular, DMN highlighted essential aspects for the description of several neuro-psychological diseases; indeed, evidence supports disruptions of DMN in Alzheimer and ASD affected subjects.

Further, BOLD signal fluctuations can be used to highlight correlations in the activity of other brain areas. For these reasons, it is crucial to define areas or region of interest and the criteria for their spatial definition. Atlas with a much more spatially detailed between can be used in addition to the DMN in the resting state fMRI analysis.

All scans presented in this paper were obtained on a single 1.5-Tesla scanner using a gradient echo-planar imaging sequence for fMRI. The fMRI data were acquired using pre-settled sequences with TR/TE 3000/50, FA 90, a DFOV $22.8 \mathrm{~cm} \times 22.8 \mathrm{~cm}$ on a matrix of $128 \times 128$, and thickness of $4 \mathrm{~mm}$.

\section{ROI and clusters-driven networking}

The first step in fMRI statistical analysis is to create a statistical map, i.e. to identify which regions are "activated" above proper statistical thresholds. How to identify these regions of Interest (ROIs)? How to look for particular ROIs related to the nature of diseases? We need to introduce some aspects of the so-called "ROI analysis" [18]. ROIs can be identified and characterised with two different strategies: 1) by functional mapping of brain regions performed, validated and verified by means of several experiments and studies of the brain (Talairach Atlas); 2) by fMRI, focusing on the activated areas, corresponding to clusters of voxels, during the performance of a task. Although the presence of some disadvantages, this approach allows for the construction of brain networks from a different point of view, i.e. from functional spontaneous clustering of the brain. ROI and clustering-guided (or led) strategies may be used to investigate the alteration introduced by "external conditions" in the brain networks. Research in functional connectivity MRI (fcMRI) has recently focused on the exploration of method taskinduced brain network, delivering significant results; further, this approach can also be applied in the rest conditions, and this is a promising pathway to get more in-depth knowledge about brain behaviours and 
performances. However, innovative mathematical methods and analysis are required to treat this information.

\section{Data analysis, connectivity measures and fcMRI: Networking the brain functions: Towards a pipeline for brain networks analysis.}

Connectivity measures may be used to test the alteration of brain connectivity in pathological occurrences. Resting state data in functional MRI (rsfMRI), as well as taskrelated designs, can be analysed with fcMRI. Different software for connectivity measures are freely available; however, the usual protocol to perform connectivity analyses comprises the following steps:

a) Functional data acquisition

Using the typical EPI MRI sequences, it is possible to collect data from experiments both with the restingstate conditions and with a task design.

b) Structural data acquisition.

To plot the functional results on the real brain anatomy of patient, anatomical MRI acquisition is performed for each subject. Also, this allows for the definition of the Grey Matter, White Matter and Cerebrospinal Fluid areas for the segmentation operation to perform noise corrections in the functional images.

c) ROI definition:

ROIs may be defined in different ways to achieve the best connections analysis model suitable for the aims of the study. It is possible to use text files with a list of the Montreal Neurological Institute (MNI) positions or a list of Talairach locations; also, on the web, there are several freely available tools for the conversion in these two References Systems (http://sprout022.sprout.yale.edu/mni2$\mathrm{tal} / \mathrm{mni}$ tal.html).

Voxels position is related to functional areas using a complete list of Brodmann areas. This fact allows for voxel-to-voxel analysis as well as "seed areas" to voxel, and ROI-to-ROI analysis of connectivity properties at the single subject level and the group level.

In all our research projects, functional connectivity toolbox Conn (www.nitrc.org/projects/conn) [19] was employed; CONN has implemented the CompCor algorithm for physiological and other noise sources reduction strategies. CONN allows for removal of movement, temporal covariates accounting, and a temporal filtering of BOLD signal oscillations. Further, Conn noise reduction strategy allows for the physiological interpretation of anticorrelations, for it does not rely on global signal regression.

In CONN software the usual fMRI preprocessing is performed: spatial preprocessing procedures include slice-timing correction, realignment, coregistration, normalisation, and spatial smoothing. In addition to these steps, the CONN software employs segmentation of Grey Matter, White Matter, and CSF areas to use during removal of temporal confounding factors. Conn spatial preprocessing is implemented by SPM software (Wellcome Department of Imaging Neuroscience, London, UK; www.fil.ion.ucl.ac.uk/spm). Both SPM and Conn run in the Matlab calculation environment (The MathWorks, Natick, Massachusetts). Regarding statistical parametric mapping, this was performed in the framework of General Linear Model (GLM) (Friston et al. 1995) as implemented by FSL (FMRIB Software Library v5.0) by the Analysis Group (FMRIB, Oxford, UK). Usual random effects procedures, as developed by Holmes and Friston [20], to describe multi-subject functional neuroimaging data for valid population inference.

To avoid confounders and limit the noise for the evaluation of the neural activity and connectivity as possible, a robust statistical analysis must address the study of noise sources in fMRI. The first step of this procedure is the denoising of the BOLD signal, aimed to define, explore, and remove possible confounders such as patient movement induced alterations. Nevertheless, it is also possible to assess the spectral contribution of all the frequencies fluctuations of the BOLD signal in both resting state, and task-driven experiments: data filtering by separating bands components allows for the contributions exploration of low or fast components of the signals.

Usually, in fcMRI, two levels of the analysis may be identified: the first level for within-subject analysis, and the second level for between-subjects analysis. Notably, the former level defines and explores functional connectivity of different kinds of sources for each subject. Successively, a second-level analysis is performed to study between-subjects contrasts of interest, hence to define population phenotypes. These two processes highlight some variables that may be defined as covariates and included in the GLM model.

As previously reported, BOLD signals from voxels and ROIs of interest can be processed in CONN software using several indices of connectivity. Connectivity measure is performed at the voxel-to-voxel level and, to discuss connectivity properties in connection with spatially segregated brain functions, a seed to voxel, and ROI to ROI analysis can be performed. Usually, brain areas of interest were spatially labelled as in Broadmann areas atlas (BA) to characterise the source regions for the extraction of the time series of interest. These areas are subsequently used as labels for targets in ROI-to-ROI analysis, and seed to voxel analysis. All Ddata presented in this paper focuses on the zero-lagged bivariatecorrelation and linear measure of functional connectivity between two sources, $x$ and $y$, defined as [19]:

Equation 1 Bivariate correlation 


$$
r=\left(x^{t} \cdot x\right)^{\frac{1}{2}} \cdot b \cdot\left(y^{t} \cdot y\right)^{-\frac{1}{2}}
$$

where.

Equation 2 Bivariate regression

$$
b=\frac{x^{t} \cdot y}{x^{t} \cdot x}
$$

This is the Voxel-Level Functional Connectivity MRI measurement derived from the Voxel-to-Voxel Connectivity Matrix $r(x, y)$; in this project, the strength of the global connectivity pattern between each voxel and the rest of the brain with the Intrinsic connectivity contrast (ICC) [21] was characterised.

Equation 3 Intrinsic connectivity contrast index

$$
C_{n}(G)=\frac{1}{|G|-1} \cdot\left|G_{n}\right|
$$

Successively, it is possible to use the "cost function" as a measure, at ROI and network level, of the links properties. In particular, for ROI-level tests:

Equation 4 Cost function of the $n$ node graph $G$

$$
C(G)=\frac{1}{|G|} \cdot \sum_{n \in G} C_{n}(G)
$$

while, for network-level measures, we can define:

Equation 5 Cost at network level

$$
I C C=\frac{1}{|\Omega|} \sum_{y \in \Omega}|r(x, y)|^{2}
$$

where $C_{n}(G)$ represents the cost in graph $G$, and $|G|$ represents the number of nodes in graph $G[19,22]$.

The word "cost" is used to define the weight property of the link, a way to determine the intensity of the link between nodes and all over the network (or net path). The weight of a path in a graph can be defined as the sum of the weights of the traversed edges.

Indices like the Betweenness Centrality, an indicator of the number of "shortest paths" for a specific node, can be used to describe some "community" properties of the nodes. A node with high betweenness centrality has a great importance in the network.

All these parameters can give us the numeric evaluation of the topological properties of the network realised by the ROIs of the brain connected by the function execution task [23].

A display of fcMRI can be achieved through several representations employing CONN toolbox for Matlab. It is possible to show the performed graph-theory analysis of the ROIs networks, characterising the topological properties of the brain networks related to the disease; the characterisation is obtained through graphics and pictures, as reported in the figures of the next paragraph.

This fact allows the researchers for several considerations about the areas involved and the functions performed by these regions. The definition of the network's organisation is related to the "self-organisation" property of the brain.

Self-organization is one of the emerging properties of the brain networks that can be related to the mechanism activated by the brain trying to solve the inefficiency associated with the action of the diseases in the brain.

This is a significant contribution arising from the application of the complex networks theory to the brain behaviour, and it refers to the so-called Networks Medicine.

\section{Applications of networks analysis: Network medicine, and network-based approach to brain diseases. Ideas and hypothesis for the use of fcMRI}

Network medicine represents the powerful tool to systematically investigate the molecular complexity of a particular disease, the molecular relationships between apparently distinct phenotypes, and the pathological alterations induced in functional brain networks [16]. The theoretical and methodological basis of this new discipline has been successfully tested in several clinical applications, and it may lead to the early identification of typical disease topology and pathways, increasing the specificity and the sensitivity of the current diagnostic methods. In this paper, some applications of this strategy/technique are proposed for the study of brain networks alterations induced in distinct phenotypes.

A first attempt to apply networks theory to fMRI signal was conducted to test the hypothesis of circuitry alterations in attention-control brain networks in TS. In particular, we can consider cortical-striatalthalamic-cortical networking alterations and the potential involvement of the frontostriatal frontoparietal circuitries. A task driven experiment was designed for a modified finger tapping motor task. Randomized commands for patients in the MRI scanner trigger the switch from usual finger tapping to the ones with avoidance of middle finger/third digit tap. Therefore, this task should stimulate different control strength on the patient that can be quickly gained by Tourette patients due to their higher control ability. Taskinduced activation or deactivation may be detected as circuitry alterations in the four major regions of interest constituting the default mode network (DMN), e.g. the posterior cingulate cortex (PCC), the cortex in the medial prefrontal region (MPFC), and left and right lateral parietal cortices (LLP and RLP). This 
scenario seems to be the proper environment for the application of Networks Theory to test the dynamic of the organisation and the properties of brain function. It is an ambitious task, especially considering that fMRI is a "noisy" low-intensity signals diagnostic method. After the characterisation of the Tourette Syndrome, a typical neurological diseases [24-26], we will present the study of the association between taste processing phenotypes and brain networks and the research about the immunological system disease and brain networks, to illustrate the connection potentiality in the diseases diagnosis process.

\section{Tourette}

One of the studies described in this article is based on the fMRI analysis of patients with TS during the performance of a complex motor task. Several studies about the TS emphasise the role of the cortical-striatal-thalamocortical brain circuits, particularly the subcortical components as the basal ganglia, and the cortical component as the prefrontal cortex [24, 25].

For this study, we acquired functional magnetic resonance imaging data from 11 individuals with Tourette's syndrome, and 11 healthy control subjects. Subjects were required to perform a complex motor task based on a switching on demand between two different conditions of finger tapping. These two different conditions required a high attention to motor strategy and a strength of maintenance this focus throughout the execution time of the task.

TS patients frequently present atypical brain connectivity [24]. However, the neural networks underlying their motor and vocal tic development are still poorly understood $[25,26]$. In particular, this experiment focused on the front-striatal system networks, which are responsible for self-regulatory control [27]. The Fronto-striatal system includes lateral inferior prefrontal cortex, medial frontal gyrus, dorsolateral prefrontal cortex, lenticular nucleus (Pallido, and Putamen), and thalamus. Fronto-striatal systems are implicated in control, and inhibition of movements, and behaviours. Based on the literature, the hypothesis of an altered network functioning, responsible for setting specific actions in response to a particular stimulus, may be a satisfactory explanation for TS clinical manifestations.

The brain areas involved in attentional executive functions are the fronto-striatal and fronto-parietal systems. The former modulates the self-regulatory control, i.e. cognitive or affective self-monitoring, and motor area. The latter regulates the so-called "adaptive control", adjusting the transition from a movement to another [15, 28].

So we proposed the submission to pathological and healthy subjects of a motion task, with higher cost function related to the higher attention requested by motion difficult; this should allow for revealing tic neural mechanisms in TS, mapping of connectivity within corticalstriatal-thalamic-cortical circuits [24, 28, 29]. Previous neuroimaging studies showed that attention task not only may stimulate/activate motion-selective cortical areas but also several others that seemed to be unrelated. Increasing or decreasing of activation in a cortical area is often attributed to attentional modulation of the cortical projections to that area. This fact leads to the idea that, in Tourette patients, attention is associated with changes in brain connectivity.

In our experiment, subjects completed a finger tapping motor task using both right and left hand and switching between the two conditions: usual finger tapping, and a finger tapping without opposition between thumb and medium fingers. Patients were not required to perform the task as quickly as possible. Participants completed a brief practice session before scanning to familiarise with stimuli and task requirements. The vocal command to switch between the two conditions was randomly submitted to increase the difficulty of the task.

All subjects were submitted to MRI analysis under the same stimulus conditions. The hypothesis is that the hemodynamic responses addressed by the attentional component of the task may reveal a cortical-striatalthalamic-cortical network alteration.

We investigated the potential frequency-modulation of fcMRI measures related to a differential actions of brain circuitries using for each condition under study a specific band-pass filter [15] Additional information may be found in Buzsaki's paper, Science 2004 [17]. The following pictures show some preliminary results: differential networks between Tourette and control subjects, with associated parameters, which measure these differences.

Interestingly, although "cost function" is higher in TS than controls, the efficiency of the differential networks is higher in pathological subjects (Fig. 1). Furthermore, the degree of the network increases with the clustering coefficient, while network path lengths are similar in the two groups. To confirm such results, population size should be increased. Nevertheless, these data suggest that brain networks analysis may be a successful strategy for the characterisation of this condition.

\section{Propylthiouracil}

From the evolutionary point of view, the ability to perceive the bitter taste of bad food, leading to the rejection of the food, may be considered a sort of surviving ability, which propels natural selection. In this way, poisonous and dangerous food can be readily detected by their bitter taste, meaning that taste has a crucial role in evolution. Indeed, nutrition plays a vital role in evolution, and in the phenotype characterisation related to the diffusion of several pathologies. Nutrition and brain processes of 
taste perception are becoming prominent aspects in the diagnosis and prognosis of several neurological diseases. Nevertheless, there are few neuroimaging studies on taste perception and processing in the brain, and some of these studies are only related to the cortical brain areas [30]. To identify the areas involved in bitter taste perception and their connectivity, a population of 6 healthy volunteers were analysed through the functional magnetic resonance imaging techniques. The sensitivity to the bitter taste of thiourea compounds, such as 6-npropylthiouracil (PROP), is a well-known example of genetic bitter taste variability. Individual differences in taste sensitivity to 6-n-propylthiouracil (PROP) may be associated with different activation patterns in the brain. Bitter taste sensitivity greatly varies among subjects and, in the case of PROP, a genetic modification related to the bitter sensitivity of the differently classified subjects was observed. For this study, the sensitive subjects group were organised and labelled as super testers, while insensitive ones, or "non-responsive", were labelled as non-tester. The term super-taster is used to distinguish individuals who perceive PROP as extremely bitter from those (medium tasters) who perceive it as moderately bitter. Several investigations attempted to elucidate molecular basis, genetic and psychophysical features of this human trait. Some studies consistently reported that PROP "supertasters" have a higher density of fungiform papillae on the anterior tongue surface, suggesting an anatomical difference in their peripheral taste system. Although human brain pathways involved in the complex gustatory circuitry are well known, it is still necessary to elucidate how the signals related to different food are processed in the brain. It is also important to establish whether such stimuli may influence different brain areas depending on the meaning that those food and taste have for each subject.

Preliminary data from functional magnetic resonance imaging (fMRI) acquired by our research group aimed at determining if individual genetic differences of taste perception of PROP can be represented in the brain by different activation patterns and connections. The four major regions of interest (ROIs) constituting the default mode network (DMN) are the medial prefrontal cortex (MPFC), the posterior cingulate cortex (PCC), the medial prefrontal, and left and right lateral parietal cortices (LLP and RLP). These regions were preliminary adopted in this study as brain activity sources for connectivity analysis. The complete set of Broadmann areas was considered as targets for connectivity analysis. Subjects underwent single fMRI run. During the scan, subjects were requested to stay still with relaxed muscles, and closed eyes. For each subject, fMRI study included 15 min recording of the brain activity at rest, followed by $15 \mathrm{~min}$ recording of the activity induced by PROP.
PROP administration occurred by placing on the tip of the tongue a filter paper disk impregnated with a $50 \mathrm{mM}$ aqueous PROP solution.

The hypothesis for this experiment was that connectivity in appetitive and aversive neural circuits may correlate to the complexity of the complex neuronal circuits.

In this framework, it is possible to generate several schemes of experiments with coherent cognitive states to guide the autonomous behaviour of the subjects. Such processes may be strictly related to deeper structures of the brain, and, in turn, related to several areas of the prefrontal cortex. Analysis of neural signalling seems to highlight the role of the amygdala and orbitofrontal cortex, two brain areas involved in taste stimuli.

It seems that a dynamic relationship exists between circuits such as enhanced arousal or attention, potentially influencing behavioural choices, e.g. food intake or style [31].

These data underline the formidable challenges ahead in describing how the food taste neural circuits interact to produce a more complex and wide range of behaviours, also confirmed by others similar study [30].

In this preliminary fMRI project, six ( 1 males, and 5 females: age $28.6 \neg \pm 0.86$ years) caucasian, non-smoker subjects were recruited according to standard procedures to perform a pilot study.

All subjects were genotyped for receptor TAS2R38 (the specific receptor of taste cells, which binds the thiocyanate group from PROP) using PCR techniques and then following the sequencing of the obtained fragments. Subjects were classified by their PROP taster status. The status was assigned through taste intensity rating in Labelled Magnitude Scale (LMS) using three suprathreshold PROP $(0.032,0.32$, and $3.2 \mathrm{mM})$ and $\mathrm{NaCl}(0.01,0.1,1.0 \mathrm{M})$ solutions. Subsequently, three subjects classified as PROP super-tasters showed homozygous genotype for taster receptor variant (PAV/PAV), while the remaining three were classified as non-tasters and showed homozygous genotype for the non-taster receptor variant (AVI/AVI), .

In this pilot study, subjects underwent one fMRI run in the scanner. For the fMRI scan, subjects followed the procedure previously described. For each subject, the fMRI exam included a 15 min recording of the brain activity at rest followed by other $15 \mathrm{~min}$ of recording of the brain activity induced by PROP bitter taste stimulation. PROP stimulation was performed by placing a filter paper disk impregnated with $50 \mathrm{mmol} / \mathrm{L}$ of the compound on the tip of the tongue. Taste stimulation induced brain activity revealed by fMRI. Interestingly, interactions between PCC and other areas of the brain were observed and reported in Fig. 2.

Considering as Source the Posterior cingulate (PCC) cortex, some correlated target areas are: 1) Target 

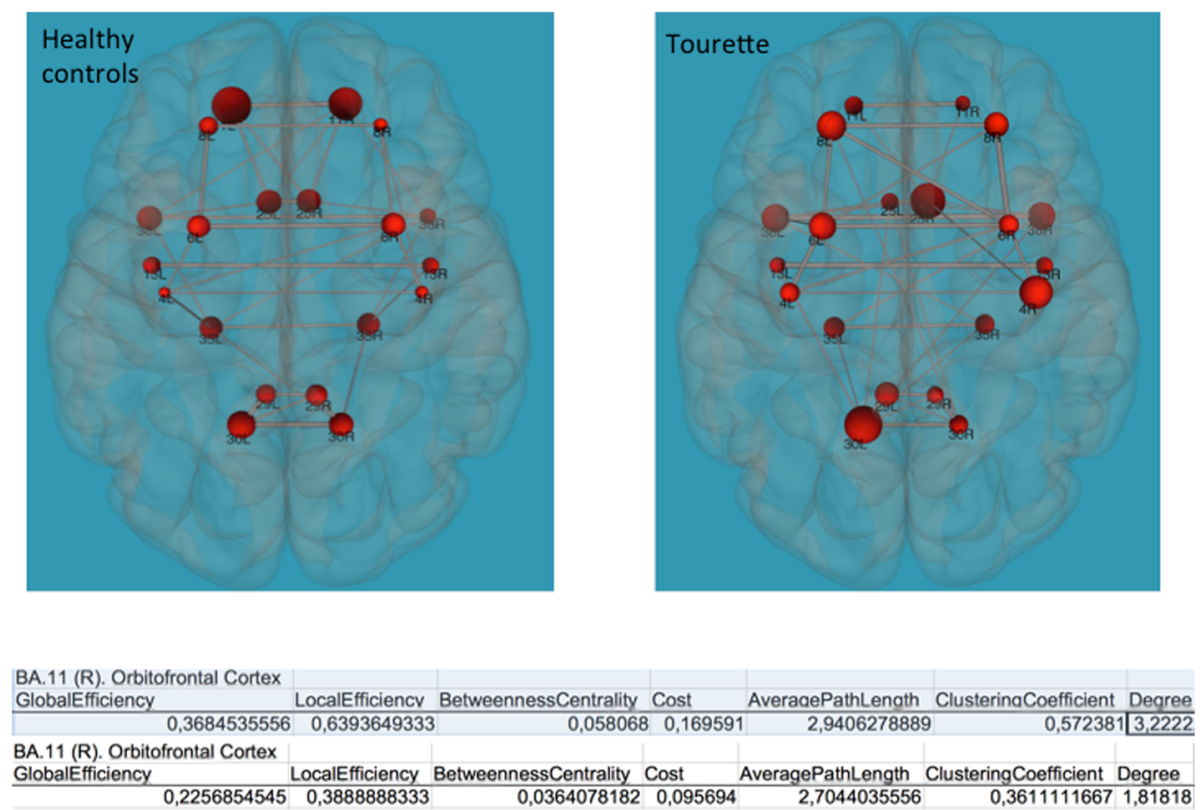

Fig. 1 Graph analysis and parameters calculated in the Tourette study

BA33(R,L): cingulate gyrus, in charge of functions as detection of errors, risk and conflict management, response inhibition, cognitive control, adaptation; an important part of the Limbic System. 2) Target BA43(R): subcentral areas with associated functions as "gustatory

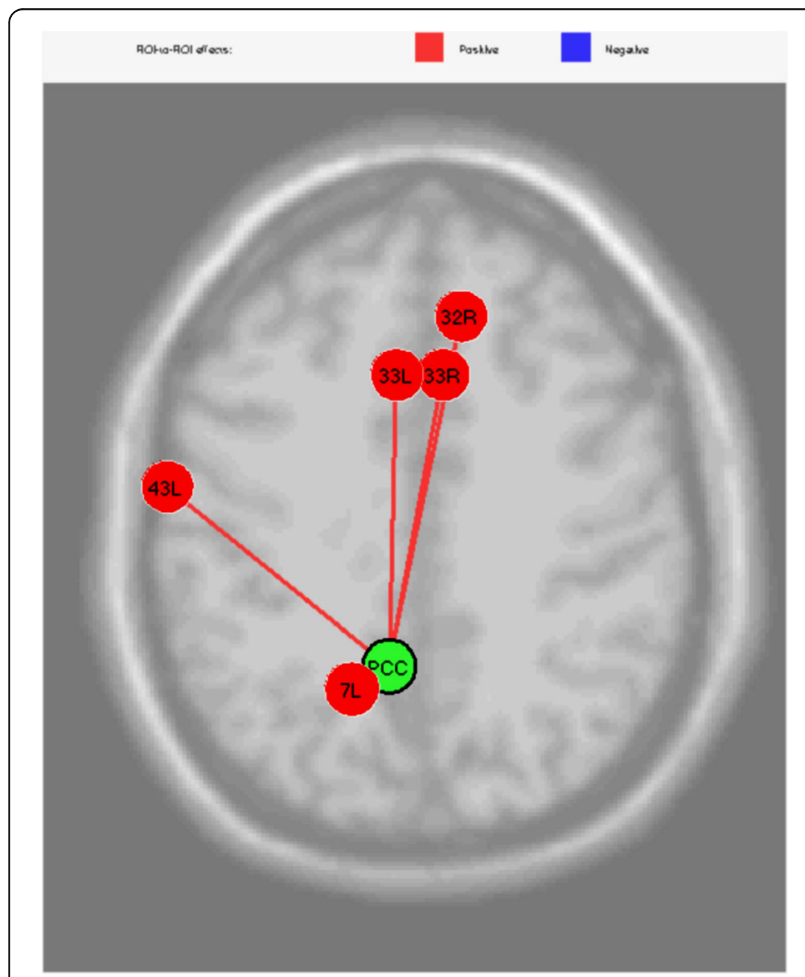

Fig. 2 PCC connections during the taste stimuli processing cortical area". 3) Target BA7(L): somatosensory associative cortex functions. 4) Target BA32(R): [15, 27, 32]. Functions: it appears to play an essential role in the regulation of the blood pressure and a wide variety of autonomic functions, such as the regulation of the heart rate. It is also involved in rational cognitive functions, such as reward anticipation, decisionmaking, empathy, impulse control, and emotion. All these functions have been related to the feeling reported by subjects after MRI examination, and to the actions activated and controlled by the brain in consideration of the requested response to the stimuli intensity experienced.

Using the Voxel to Voxel mapping for correlations representation it is possible to generate an SPM [20]style picture of direct and inverse correlation between activated Voxels, as reported in Fig. 3:

Using this representation it can be observed that the activation of LLP source was inversely correlated with Premotor Cortex (left), Dorsolateral Prefrontal Cortex (right) and Retrosplenial Cingulate Cortex (right), and directly with Primary Visual Cortex V1 (left). The MPFC source was inversely correlated with Somatosensory Associative Cortex (right) and Anterior Entorhinal Cortex (right). The PCC source was directly correlated to the Anterior Cingulate Cortex (right), Anterior Cingulate Cortex (left), Subcentral Area (right), Somatosensory Associative Cortex (left) and Dorsal Anterior Cingulate Cortex (right). The RLP source was directly correlated with Auditory Cortex (right) and Insula Cortex (left) [16, 17, 28]. 


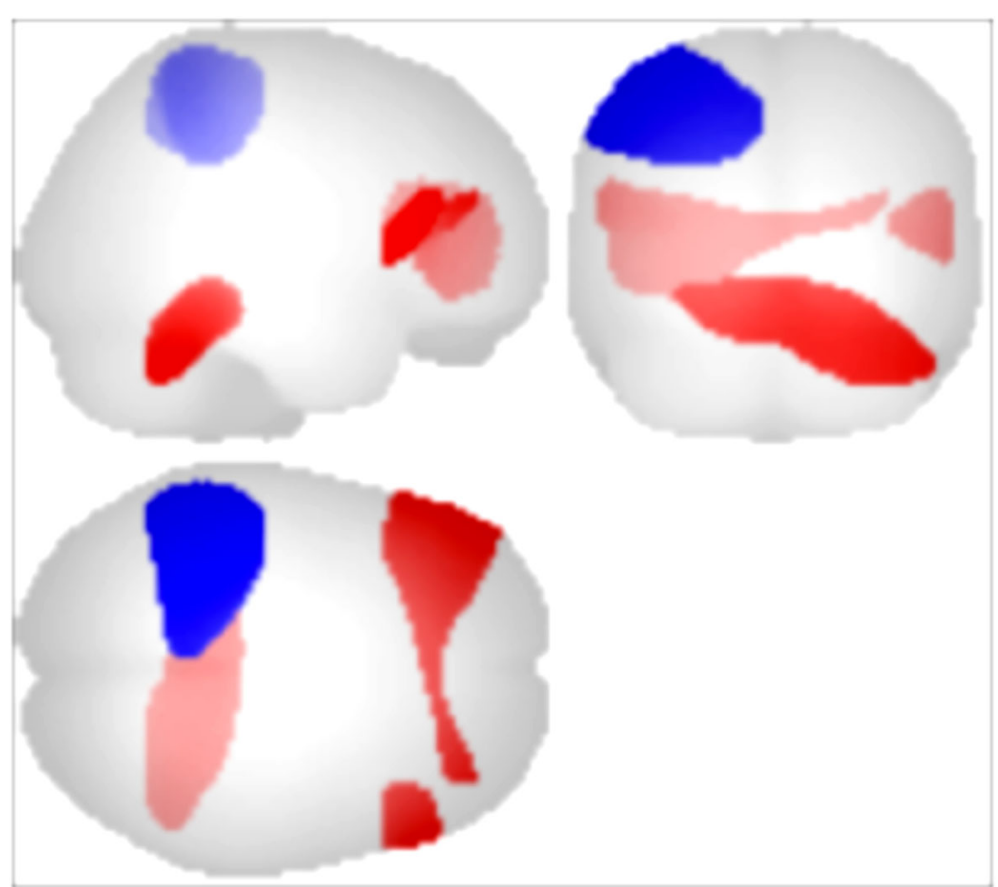

Fig. 3 Direct and inverse correlated areas of the brain during the task execution in analysed population

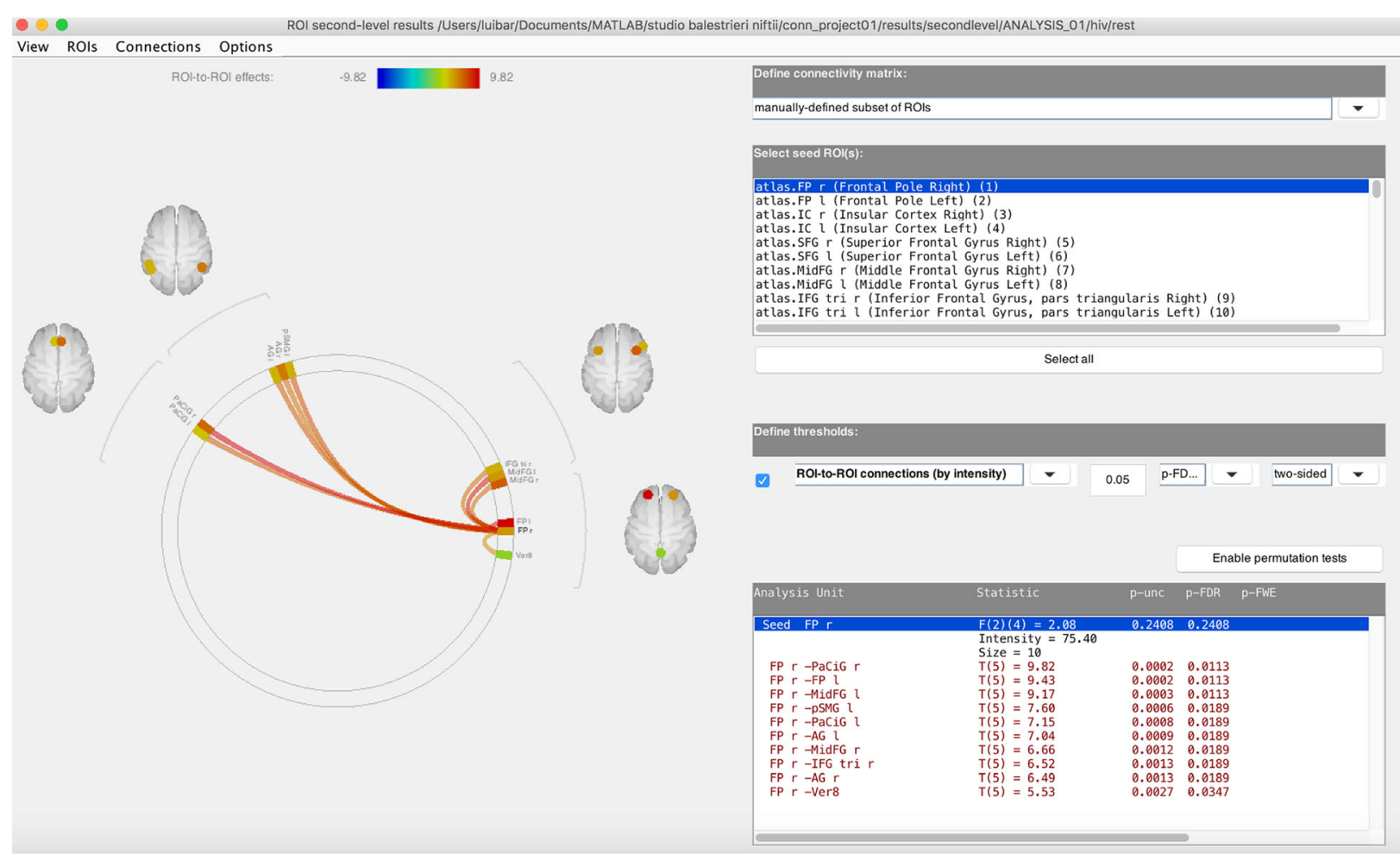

Fig. 4 HIV monoinfected 


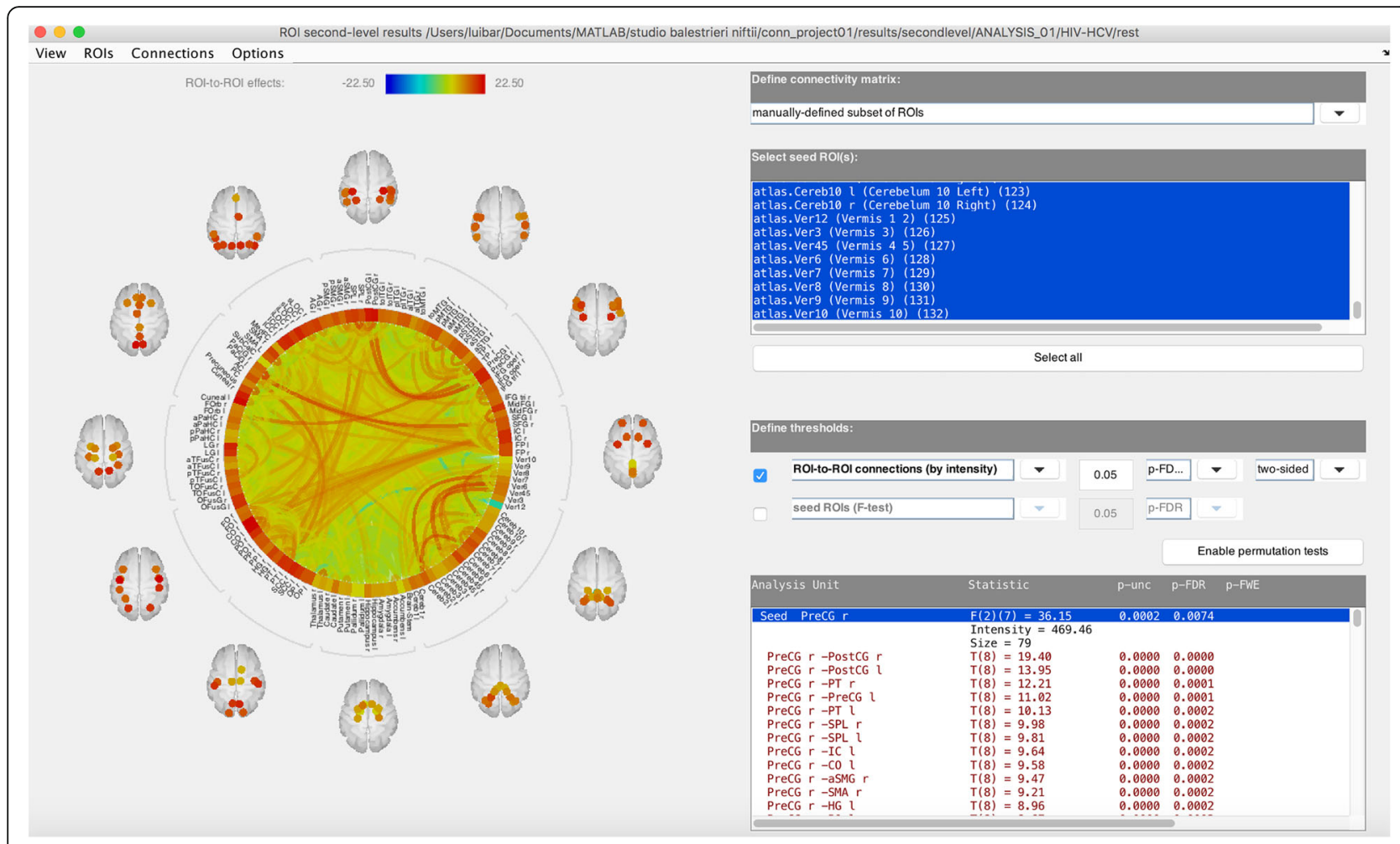

Fig. 5 HIV-HCV coinfected

In conclusion, preliminary results showed the different Cortical Areas in which PROP bitter taste signals are differently processed by the two genetic groups (PROP super-taster PAV/PAV subjects, and non-taster AVI/AVI subjects).

These preliminary results are consistent with individual differences in the ability to taste this compound, and highlight differences in neuronal connectivity indicating that super-tasters and non-tasters may also differ at the central level, and not only at the periphery. Further analysis with a larger population should be performed: nevertheless, this seems to be an exciting insight on eating behaviour and the food intake at brain level in humans [33, 34].

\section{HIV-HCV study}

The management of patients infected with hepatitis $\mathrm{C}$ virus (HCV) depends on comorbidity factors, and different phenotypes can have different disease progression. Data from studies on human immunodeficiency virus (HIV) comorbidity suggest that HIV/ $\mathrm{HCV}$-coinfected patients have different progress diseases compared to HCV-monoinfected patients. In fact, progression rate increases with older age, alcoholism, male sex, and HIV co-infection, with a higher risk of cirrhosis or decompensated liver than
HCV-monoinfected patients $[35,36]$. Moreover, coinfected patients report cognitive alterations, and it is not evident the contributions of the single infections. Preliminary fcMRI data were collected from an undergoing study in our Research Unit to estimate the contribution of each kind of infection on drug activity against cognitive decay.

Although a limited number of patients was enrolled for this study, we observed different brain connectivity mapping between HIV monoinfected and HIV-HCV coinfected. These differences were found in areas not included in the DMN (MPFC, PCC, LLP and RLP), but in some other areas classified in the Atlas present in the CONN Toolbox.

Perturbations seem to be statistically stronger in other areas than DMN's ones. We report these activations to prove the sensibility of the method to study the alterations induced in the brain by immunological diseases.

The comparison of connectivity between HIV monoinfected and HIV-HCV coinfected can be compatible with a different brain networking of subjects in the groups (Figs. 4 and 5). It means that different brain areas can be involved in these two populations considering the differences in the cognitive, motor and somatosensory alterations. fcMRI can reveal these variations leading to a more focused and tailored therapy for the subjects. 


\section{Conclusions}

The fc-MRI analysis offers a standard framework to perform a broad set of connectivity-based experiments on brain functions under a variety of pathological conditions. Calculation of several connectivity parameters and graph theory measurements for both resting state and task fMRI allow for the investigation of brain reactions/responses to different pathological alterations. Although preliminary data, the reported results show three examples of high reliability for different fcMRI measure conditions, suggesting their potential application as useful clinical practice.

\section{Acknowledgements}

Authors wish to thank Diana Caravana, Paolo Contu and Franco Conti, Technicians in MRI at the Department of Radiology - University of Cagliari AOU - Cagliari - Italy, for their contribution and expertise in the performing of the exams.

\section{Availability of data and material}

(please contact directly the corresponding author).

\section{Funding}

No source of funding to declare.

\section{Author's contributions}

LB performed the data analysis, was a major contributor in writing the manuscript. FM participated in the design of the study, analyzed and interpreted the data regarding the TOURETTE disease. ITB participated in the design of the study, analyzed and interpreted the data regarding the PROP test and was a contributor in the writing manuscript. MM participated in the design of the study, analyzed and interpreted the data regarding the PROP test. HSS participated in the design of the paper, and was a contributor in the writing manuscript. AM analyzed and interpreted the data regarding the HIV vs HIV-HCV study. JSS participated in the design of the paper, and was a contributor in the writing manuscript. $A B$ performed the MRI examination of the HIV vs HIV-HCV study. MA suggested improvements in the MRI protocols applied. LS performed the MRI examination and was a contributor in the manuscript writing. All authors read and approved the final manuscript.

\section{Authors' information}

Not applicable.

\section{Ethics approval and consent to participate}

(Not applicable).

\section{Consent for publication}

(Not applicable).

\section{Competing interests}

The authors declare that they have no competing interests.

\section{Publisher's Note}

Springer Nature remains neutral with regard to jurisdictional claims in published maps and institutional affiliations.

\section{Author details}

${ }^{1}$ Department of Medical Sciences and Public Health, University of Cagliari, Cagliari, Italy. ${ }^{2}$ Department of Nuclear Medicine, Universitary Hospital of Cagliari AOU, Cagliari, Italy. ${ }^{3}$ Department of Biomedical Sciences, University of Cagliari, Cagliari, Italy. ${ }^{4}$ Diagnosis and Monitoring Division, AtheroPoint LLC, Roseville, CA, USA. ${ }^{5}$ Department of Radiology, University of Cagliari AOU, Cagliari, Italy. "Department of Radiology, University of Rome "La Sapienza", Rome, Italy.
Received: 20 December 2016 Accepted: 23 October 2017

Published online: 10 November 2017

\section{References}

1. Albert-Laszlo Parabasi, "Network Science by Albert-László Barabási." [Online]. Available: http://barabasi.com/networksciencebook/.

2. J. C. Gore, "Principles and practice of functional MRI of the human brain.," J Clin Invest, Jul. 2003, vol. 112, no. 1, pp. 4-9.

3. X. Hu and E. Yacoub, "The story of the initial dip in fMRI.," Neurolmage, Aug. 2012, vol. 62, no. 2, pp. 1103-1108.

4. Logothetis NK, Auguth M, Oeltermann A, Pauls J, Trinath T. A neurophysiological investigation of the basis of the BOLD signal in fMRI. Nature. 2001:412(6843):150-7.

5. H. Yuan, C. Perdoni, L. Yang, and B. He, "Differential electrophysiological coupling for positive and negative BOLD responses during unilateral hand movements.," J Neurosci, Jun. 2011, vol. 31, no. 26, pp. 9585-9593.

6. Saba L. Magnetic Resonance Imaging Handbook. 2016. CRC Press; ISBN 9781482216288

7. Bernstein MA, King KF, ZHOU XJ, Ehman RL, Bernstein MA, King KF, ZHOU XJ. Handbook of MRI pulse sequences. CRC Press Taylors and Francis Group; 2004. p. 1017. eBook ISBN: 9780080533124

8. S. Haller and A. J. Bartsch, "Pitfalls in fMRI," Eur Radiol, Nov. 2009, vol. 19, no. 11, pp. 2689-2706.

9. Friston KJ. Functional and effective connectivity in neuroimaging: a synthesis. Hum Brain Mapp. 1994;2:56-78.

10. K. J. Friston, "Functional and effective connectivity: a review," Brain Connect, vol. 1, no. 1, pp. 13-36, Jan. 2011

11. J. A. Detre, H. Rao, D. J. J. Wang, Y. F. Chen, and Z. Wang, "Applications of arterial spin labeled MRI in the brain.," J Magn Reson Imaging, vol. 35, no. 5, pp. 1026-1037, May 2012.

12. Kiviniemi V, Kantola JH, Jauhiainen J, Tervonen O. Comparison of methods for detecting nondeterministic BOLD fluctuation in fMRI. Magn Reson Imaging. 2004;22(2):197-203.

13. Biswal B, Zerrin Yetkin F, Haughton VM, Hyde JS. Functional Connectivity in the Motor Cortex of Resting Human Brain Using Echo-Planar MRI. Magn Reson Med. 1995;34(4):537-41.

14. Greicius MD, Krasnow B, Reiss AL, Menon V. Functional connectivity in the resting brain: a network analysis of the default mode hypothesis. Proc Natl Acad Sci U S A. 2003;100(1):253-8.

15. N. U. F. Dosenbach, D. A. Fair, F. M. Miezin, A. L. Cohen, K. K. Wenger, R. A. T. Dosenbach, M. D. Fox, A. Z. Snyder, J. L. Vincent, M. E. Raichle, B. L. Schlaggar, and S. E. Petersen, "Distinct brain networks for adaptive and stable task control in humans.," Proc Natl Acad Sci U S A, vol. 104, no. 26, pp. 11073-11078, Jun. 2007.

16. De La Fuente A, Xia S, Branch C, Li X. A review of attention-deficit/ hyperactivity disorder from the perspective of brain networks. Front Hum Neurosci. 2013;7:192.

17. G. Buzsáki and A. Draguhn, "Neuronal oscillations in cortical networks.," Science, vol. 304, no. 5679, pp. 1926-1929, Jun. 2004.

18. R. A. Poldrack, "Region of interest analysis for fMRI.," Soc Cogn Affect Neurosci, vol. 2, no. 1, pp. 67-70, Mar. 2007.

19. Whitfield-Gabrieli S, Nieto-Castanon A. Conn: a functional connectivity toolbox for correlated and Anticorrelated brain networks. BRAIN Connect. 2012:3

20. Friston KJ, Holmes AP, Worsley KJ, Poline J-P, Frith CD, Frackowiak RSJ. Statistical parametric maps in functional imaging: a general linear approach. Hum Brain Mapp, vol. 1995:2.

21. R. Martuzzi, R. Ramani, M. Qiu, X. Shen, X. Papademetris, and R. T. Constable, "A whole-brain voxel based measure of intrinsic connectivity contrast reveals local changes in tissue connectivity with anesthetic without a priori assumptions on thresholds or regions of interest.," Neurolmage, vol. 58, no. 4, pp. 1044-1050, Oct. 2011.

22. Achard S, Bullmore E. "Efficiency and cost of economical brain functional networks," PLoS Comput. Biol. 2007;3(2):e17.

23. A.-L. Barabási, N. Gulbahce, and J. Loscalzo, "Network medicine: a networkbased approach to human disease.," Nat Rev Genet, vol. 12, no. 1, pp. 5668, Jan. 2011.

24. E. Stern, D. A. Silbersweig, K.-Y. Chee, A. Holmes, M. M. Robertson, M. Trimble, C. D. Frith, R. S. J. Frackowiak, "A functional Neuroanatomy of tics in Tourette syndrome," Arch Gen Psychiatry, vol. 57, no. 8, p. 741, Aug. 2000. 
25. Z. Wang, T. V Maia, R. Marsh, T. Colibazzi, A. Gerber, and B. S. Peterson, "The neural circuits that generate tics in Tourette's syndrome.," Am J Psychiatry, vol. 168, no. 12, pp. 1326-1337, Dec. 2011.

26. A. Lerner, A. Bagic, E. A. Boudreau, T. Hanakawa, F. Pagan, Z. Mari, W. Bara-Jimenez, M. Aksu, G. Garraux, J. M. Simmons, S. Sato, D. L. Murphy, and M. Hallett, "Neuroimaging of neuronal circuits involved in tic generation in patients with Tourette syndrome," Neurology, vol. 68, no. 23, pp. 1979-1987, Jun. 2007.

27. N. Mol Debes, M. Preel, and L. Skov, "Functional neuroimaging in Tourette syndrome: recent perspectives," Neurosci Neuroeconomics, vol Volume 6, pp. 1-13, Apr. 2017.

28. J. A. Church, D. A. Fair, N. U. F. Dosenbach, A. L. Cohen, F. M. Miezin, S. E. Petersen, and B. L. Schlaggar, "Control networks in paediatric Tourette syndrome show immature and anomalous patterns of functional connectivity." Brain, vol. 132, no. Pt 1, pp. 225-238, Jan. 2009.

29. S. Bohlhalter, A. Goldfine, S. Matteson, G. Garraux, "Neural correlates of tic generation in Tourette syndrome: an event-related functional MRI study.," Brain, vol. 129, no. Pt 8, pp. 2029-2037, Aug. 2006.

30. S. Bembich, C. Lanzara, A. Clarici, S. Demarini, B. J. Tepper, P. Gasparini, and D. L. Grasso, "Individual differences in prefrontal cortex activity during perception of bitter taste using fNIRS methodology." Chem Senses, vol. 35, no. 9, pp. 801-812, Nov. 2010.

31. E. R. Grimm and N. I. Steinle, "Genetics of eating behavior: established and emerging concepts.," Nutr Rev, vol. 69, no. 1, pp. 52-60, Jan. 2011.

32. Roth RM, Saykin AJ, Flashman LA, Pixley HS, West JD, Mamourian AC. Eventrelated functional magnetic resonance imaging of response inhibition in obsessive-compulsive disorder. Biol Psychiatry. 2007;62(8):901-9.

33. V. B. Duffy, A. C. Davidson, J. R. Kidd, K. K. Kidd, W. C. Speed, A. J. Pakstis, D. R. Reed, D. J. Snyder, and L. M. Bartoshuk, "Bitter receptor gene (TAS2R38), 6-n-Propylthiouracil (PROP) bitterness and alcohol intake," Alcohol Clin Exp Res, vol. 28, no. 11, pp. 1629-1637, Nov. 2004.

34. T. Iole and L. Barberini, "Abstracts from the 24th annual meeting of the European chemoreception research organization (ECRO 2014), Dijon, France, September 10-13th, 2014," Chem Senses, vol. 40, no. 3, pp. 211-297, Mar. 2015.

35. L. Aranzabal, J. L. Casado, J. Moya, C. Quereda, S. Diz, A. Moreno, L. Moreno, A. Antela, M. J. Perez-Elias, F. Dronda, A. Marin, F. Hernandez-Ranz, A. Moreno, and S. Moreno, "Influence of liver fibrosis on highly active antiretroviral therapyassociated hepatotoxicity in patients with HIV and hepatitis $C$ virus Coinfection," Clin Infect Dis, vol. 40, no. 4, pp. 588-593, Feb. 2005.

36. P. Labarga, V. Soriano, M. E. Vispo, J. Pinilla, L. Martín-Carbonero, C. Castellares, R. Casado, I. Maida, P. García-Gascó, and P. Barreiro, "Hepatotoxicity of antiretroviral drugs is reduced after successful treatment of chronic hepatitis C in HIV-infected patients," J Infect Dis, vol. 196, no. 5, pp. 670-676, Sep. 2007.

\section{Submit your next manuscript to BioMed Central and we will help you at every step:}

- We accept pre-submission inquiries

- Our selector tool helps you to find the most relevant journal

- We provide round the clock customer support

- Convenient online submission

- Thorough peer review

- Inclusion in PubMed and all major indexing services

- Maximum visibility for your research

Submit your manuscript at www.biomedcentral.com/submit 